DESY 05-225

ISSN 0418-9833

December 2005

\title{
Measurement of Event Shape Variables in Deep-Inelastic Scattering at HERA
}

\author{
H1 Collaboration
}

\begin{abstract}
Deep-inelastic ep scattering data taken with the H1 detector at HERA and corresponding to an integrated luminosity of $106 \mathrm{pb}^{-1}$ are used to study the differential distributions of event shape variables. These include thrust, jet broadening, jet mass and the $C$-parameter. The four-momentum transfer $Q$ is taken to be the relevant energy scale and ranges between $14 \mathrm{GeV}$ and $200 \mathrm{GeV}$. The event shape distributions are compared with perturbative QCD predictions, which include resummed contributions and analytical power law corrections, the latter accounting for non-perturbative hadronisation effects. The data clearly exhibit the running of the strong coupling $\alpha_{s}(Q)$ and are consistent with a universal power correction parameter $\alpha_{0}$ for all event shape variables. A combined QCD fit using all event shape variables yields $\alpha_{s}\left(m_{Z}\right)=0.1198 \pm 0.0013_{-0.0043}^{+0.0056}$ and $\alpha_{0}=0.476 \pm 0.008_{-0.059}^{+0.018}$.
\end{abstract}

Submitted to Eur. Phys. J. C 


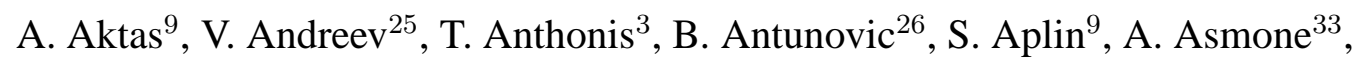

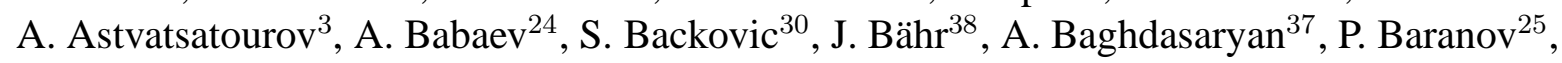

E. Barrelet ${ }^{29}$, W. Bartel ${ }^{9}$, S. Baudrand ${ }^{27}$, S. Baumgartner ${ }^{39}$, J. Becker ${ }^{40}$, M. Beckingham ${ }^{9}$,

O. Behnke ${ }^{9}$, O. Behrendt ${ }^{6}$, A. Belousov ${ }^{25}$, Ch. Berger ${ }^{1}$, N. Berger ${ }^{39}$, J.C. Bizot ${ }^{27}$,

M.-O. Boenig ${ }^{6}$, V. Boudry ${ }^{28}$, J. Bracinik ${ }^{26}$, G. Brandt ${ }^{12}$, V. Brisson ${ }^{27}$, D. Bruncko ${ }^{15}$,

F.W. Büsser ${ }^{10}$, A. Bunyatyan ${ }^{11,37}$, G. Buschhorn ${ }^{26}$, L. Bystritskaya ${ }^{24}$, A.J. Campbell ${ }^{9}$,

F. Cassol-Brunner ${ }^{21}$, K. Cerny ${ }^{32}$, V. Cerny ${ }^{15,46}$, V. Chekelian ${ }^{26}$, J.G. Contreras ${ }^{22}$,

J.A. Coughlan ${ }^{4}$, B.E. Cox $^{20}$, G. Cozzika ${ }^{8}$, J. Cvach ${ }^{31}$, J.B. Dainton ${ }^{17}$, W.D. Dau ${ }^{14}$,

K. Daum ${ }^{36,42}$, Y. de Boer ${ }^{24}$, B. Delcourt ${ }^{27}$, M. Del Degan ${ }^{39}$, A. De Roeck ${ }^{9,44}$, K. Desch ${ }^{10}$,

E.A. De Wolf ${ }^{3}$, C. Diaconu ${ }^{21}$, V. Dodonov ${ }^{11}$, A. Dubak ${ }^{30,45}$, G. Eckerlin ${ }^{9}$, V. Efremenko ${ }^{24}$,

S. Egli ${ }^{35}$, R. Eichler ${ }^{35}$, F. Eisele ${ }^{12}$, E. Elsen ${ }^{9}$, W. Erdmann ${ }^{39}$, S. Essenov ${ }^{24}$, A. Falkewicz ${ }^{5}$,

P.J.W. Faulkner ${ }^{2}$, L. Favart ${ }^{3}$, A. Fedotov ${ }^{24}$, R. Felst ${ }^{9}$, J. Feltesse ${ }^{8}$, J. Ferencei ${ }^{15}$, L. Finke ${ }^{10}$,

M. Fleischer ${ }^{9}$, P. Fleischmann ${ }^{9}$, G. Flucke ${ }^{33}$, A. Fomenko ${ }^{25}$, I. Foresti ${ }^{40}$, G. Franke ${ }^{9}$,

T. Frisson ${ }^{28}$, E. Gabathuler ${ }^{17}$, E. Garutti ${ }^{9}$, J. Gayler ${ }^{9}$, C. Gerlich ${ }^{12}$, S. Ghazaryan ${ }^{37}$,

S. Ginzburgskaya ${ }^{24}$, A. Glazov ${ }^{9}$, I. Glushkov ${ }^{38}$, L. Goerlich ${ }^{5}$, M. Goettlich ${ }^{9}$, N. Gogitidze ${ }^{25}$,

S. Gorbounov ${ }^{38}$, C. Goyon ${ }^{21}$, C. Grab ${ }^{39}$, T. Greenshaw ${ }^{17}$, M. Gregori ${ }^{18}$, B.R. Grell ${ }^{9}$,

G. Grindhammer ${ }^{26}$, C. Gwilliam ${ }^{20}$, D. Haidt ${ }^{9}$, L. Hajduk ${ }^{5}$, M. Hansson ${ }^{19}$, G. Heinzelmann ${ }^{10}$,

R.C.W. Henderson ${ }^{16}$, H. Henschel ${ }^{38}$, G. Herrera ${ }^{23}$, M. Hildebrandt ${ }^{35}$, K.H. Hiller ${ }^{38}$,

D. Hoffmann ${ }^{21}$, R. Horisberger ${ }^{35}$, A. Hovhannisyan ${ }^{37}$, T. Hreus ${ }^{15}$, S. Hussain ${ }^{18}$,

M. Ibbotson ${ }^{20}$, M. Ismail ${ }^{20}$, M. Jacquet ${ }^{27}$, L. Janauschek ${ }^{26}$, X. Janssen ${ }^{9}$, V. Jemanov ${ }^{10}$,

L. Jönsson ${ }^{19}$, D.P. Johnson ${ }^{3}$, A.W. Jung ${ }^{13}$, H. Jung ${ }^{19,9}$, M. Kapichine ${ }^{7}$, J. Katzy ${ }^{9}$,

I.R. Kenyon ${ }^{2}$, C. Kiesling ${ }^{26}$, M. Klein ${ }^{38}$, C. Kleinwort ${ }^{9}$, T. Klimkovich ${ }^{9}$, T. Kluge ${ }^{9}$, G. Knies ${ }^{9}$,

A. Knutsson ${ }^{19}$, V. Korbel ${ }^{9}$, P. Kostka ${ }^{38}$, K. Krastev ${ }^{9}$, J. Kretzschmar ${ }^{38}$, A. Kropivnitskaya ${ }^{24}$,

K. Krüger ${ }^{13}$, J. Kückens ${ }^{9}$, M.P.J. Landon ${ }^{18}$, W. Lange ${ }^{38}$, T. Laštovička ${ }^{38,32}$,

G. Laštovička-Medin ${ }^{30}$, P. Laycock ${ }^{17}$, A. Lebedev ${ }^{25}$, G. Leibenguth ${ }^{39}$, V. Lendermann ${ }^{13}$,

S. Levonian ${ }^{9}$, L. Lindfeld ${ }^{40}$, K. Lipka ${ }^{38}$, A. Liptaj ${ }^{26}$, B. List ${ }^{39}$, J. List ${ }^{10}$, E. Lobodzinska ${ }^{38,5}$,

N. Loktionova ${ }^{25}$, R. Lopez-Fernandez ${ }^{23}$, V. Lubimov ${ }^{24}$, A.-I. Lucaci-Timoce ${ }^{9}$, H. Lueders ${ }^{10}$,

D. Lüke ${ }^{6,9}$, T. Lux $^{10}$, L. Lytkin ${ }^{11}$, A. Makankine ${ }^{7}$, N. Malden ${ }^{20}$, E. Malinovski $^{25}$,

S. Mangano ${ }^{39}$, P. Marage ${ }^{3}$, R. Marshall ${ }^{20}$, M. Martisikova ${ }^{9}$, H.-U. Martyn ${ }^{1}$, S.J. Maxfield ${ }^{17}$,

D. Meer ${ }^{39}$, A. Mehta ${ }^{17}$, K. Meier ${ }^{13}$, A.B. Meyer ${ }^{9}$, H. Meyer ${ }^{36}$, J. Meyer ${ }^{9}$, V. Michels ${ }^{9}$,

S. Mikocki ${ }^{5}$, I. Milcewicz-Mika ${ }^{5}$, D. Milstead ${ }^{17}$, D. Mladenov ${ }^{34}$, A. Mohamed ${ }^{17}$, F. Moreau ${ }^{28}$,

A. Morozov ${ }^{7}$, J.V. Morris ${ }^{4}$, M.U. Mozer ${ }^{12}$, K. Müller ${ }^{40}$, P. Murín ${ }^{15,43}$, K. Nankov ${ }^{34}$,

B. Naroska ${ }^{10}$, Th. Naumann ${ }^{38}$, P.R. Newman ${ }^{2}$, C. Niebuhr ${ }^{9}$, A. Nikiforov ${ }^{26}$, G. Nowak ${ }^{5}$,

M. Nozicka ${ }^{32}$, R. Oganezov ${ }^{37}$, B. Olivier ${ }^{26}$, J.E. Olsson ${ }^{9}$, S. Osman ${ }^{19}$, D. Ozerov ${ }^{24}$,

V. Palichik ${ }^{7}$, I. Panagoulias ${ }^{9}$, T. Papadopoulou ${ }^{9}$, C. Pascaud ${ }^{27}$, G.D. Patel ${ }^{17}$, H. Peng ${ }^{9}$,

E. Perez ${ }^{8}$, D. Perez-Astudillo ${ }^{22}$, A. Perieanu ${ }^{9}$, A. Petrukhin ${ }^{24}$, D. Pitzl ${ }^{9}$, R. Plačakyté ${ }^{26}$,

B. Portheault ${ }^{27}$, B. Povh ${ }^{11}$, P. Prideaux ${ }^{17}$, A.J. Rahmat ${ }^{17}$, N. Raicevic ${ }^{30}$, P. Reimer ${ }^{31}$,

A. Rimmer ${ }^{17}$, C. Risler ${ }^{9}$, E. Rizvi ${ }^{18}$, P. Robmann ${ }^{40}$, B. Roland ${ }^{3}$, R. Roosen ${ }^{3}$, A. Rostovtsev ${ }^{24}$,

Z. Rurikova ${ }^{26}$, S. Rusakov ${ }^{25}$, F. Salvaire ${ }^{10}$, D.P.C. Sankey ${ }^{4}$, E. Sauvan ${ }^{21}$, S. Schätzel $^{9}$,

S. Schmidt ${ }^{9}$, S. Schmitt ${ }^{9}$, C. Schmitz ${ }^{40}$, L. Schoeffel ${ }^{8}$, A. Schöning ${ }^{39}$, H.-C. Schultz-Coulon ${ }^{13}$,

K. Sedlák ${ }^{31}$, F. Sefkow ${ }^{9}$, R.N. Shaw-West ${ }^{2}$, I. Sheviakov ${ }^{25}$, L.N. Shtarkov ${ }^{25}$, T. Sloan ${ }^{16}$,

P. Smirnov ${ }^{25}$, Y. Soloviev ${ }^{25}$, D. South ${ }^{9}$, V. Spaskov ${ }^{7}$, A. Specka ${ }^{28}$, M. Steder ${ }^{9}$, B. Stella ${ }^{33}$,

J. Stiewe ${ }^{13}$, I. Strauch ${ }^{9}$, U. Straumann ${ }^{40}$, D. Sunar ${ }^{3}$, V. Tchoulakov ${ }^{7}$, G. Thompson ${ }^{18}$,

P.D. Thompson ${ }^{2}$, F. Tomasz ${ }^{15}$, D. Traynor ${ }^{18}$, P. Truöl ${ }^{40}$, I. Tsakov ${ }^{34}$, G. Tsipolitis ${ }^{9,41}$,

I. Tsurin ${ }^{9}$, J. Turnau ${ }^{5}$, E. Tzamariudaki ${ }^{26}$, K. Urban ${ }^{13}$, M. Urban ${ }^{40}$, A. Usik ${ }^{25}$, D. Utkin ${ }^{24}$,

A. Valkárováa ${ }^{32}$, C. Vallée ${ }^{21}$, P. Van Mechelen ${ }^{3}$, A. Vargas Trevino ${ }^{6}$, Y. Vazdik ${ }^{25}$, C. Veelken ${ }^{17}$, 
S. Vinokurova ${ }^{9}$, V. Volchinski ${ }^{37}$, K. Wacker ${ }^{6}$, J. Wagner $^{9}$, G. Weber ${ }^{10}$, R. Weber $^{39}$,

D. Wegener ${ }^{6}$, C. Werner ${ }^{12}$, M. Wessels ${ }^{9}$, B. Wessling ${ }^{9}$, C. Wigmore ${ }^{2}$, Ch. Wissing $^{6}$, R. Wolf $^{12}$,

E. Wünsch ${ }^{9}$, S. Xella ${ }^{40}$, W. Yan ${ }^{9}$, V. Yeganov ${ }^{37}$, J. Žáček ${ }^{32}$, J. Zálešák ${ }^{31}$, Z. Zhang ${ }^{27}$,

A. Zhelezov ${ }^{24}$, A. Zhokin ${ }^{24}$, Y.C. Zhu ${ }^{9}$, J. Zimmermann ${ }^{26}$, T. Zimmermann ${ }^{39}$, H. Zohrabyan ${ }^{37}$, and F. Zomer ${ }^{27}$

${ }^{1}$ I. Physikalisches Institut der RWTH, Aachen, Germany ${ }^{a}$

${ }^{2}$ School of Physics and Astronomy, University of Birmingham, Birmingham, $U K^{b}$

${ }^{3}$ Inter-University Institute for High Energies ULB-VUB, Brussels; Universiteit Antwerpen,

Antwerpen; Belgium ${ }^{c}$

${ }^{4}$ Rutherford Appleton Laboratory, Chilton, Didcot, $U K^{b}$

${ }^{5}$ Institute for Nuclear Physics, Cracow, Poland ${ }^{d}$

${ }^{6}$ Institut für Physik, Universität Dortmund, Dortmund, Germany ${ }^{a}$

${ }^{7}$ Joint Institute for Nuclear Research, Dubna, Russia

${ }^{8}$ CEA, DSM/DAPNIA, CE-Saclay, Gif-sur-Yvette, France

${ }^{9}$ DESY, Hamburg, Germany

${ }^{10}$ Institut für Experimentalphysik, Universität Hamburg, Hamburg, Germany ${ }^{a}$

${ }^{11}$ Max-Planck-Institut für Kernphysik, Heidelberg, Germany

${ }^{12}$ Physikalisches Institut, Universität Heidelberg, Heidelberg, Germany ${ }^{a}$

${ }^{13}$ Kirchhoff-Institut für Physik, Universität Heidelberg, Heidelberg, Germany ${ }^{a}$

${ }^{14}$ Institut für Experimentelle und Angewandte Physik, Universität Kiel, Kiel, Germany

${ }^{15}$ Institute of Experimental Physics, Slovak Academy of Sciences, Košice, Slovak Republic ${ }^{f}$

${ }^{16}$ Department of Physics, University of Lancaster, Lancaster, $U K^{b}$

${ }^{17}$ Department of Physics, University of Liverpool, Liverpool, $U K^{b}$

${ }^{18}$ Queen Mary and Westfield College, London, $U K^{b}$

${ }^{19}$ Physics Department, University of Lund, Lund, Sweden ${ }^{g}$

${ }^{20}$ Physics Department, University of Manchester, Manchester, $U K^{b}$

${ }^{21}$ CPPM, CNRS/IN2P3 - Univ. Mediterranee, Marseille - France

${ }^{22}$ Departamento de Fisica Aplicada, CINVESTAV, Mérida, Yucatán, México ${ }^{j}$

${ }^{23}$ Departamento de Fisica, CINVESTAV, México ${ }^{j}$

${ }^{24}$ Institute for Theoretical and Experimental Physics, Moscow, Russia ${ }^{k}$

${ }^{25}$ Lebedev Physical Institute, Moscow, Russia ${ }$

${ }^{26}$ Max-Planck-Institut für Physik, München, Germany

${ }^{27}$ LAL, Université de Paris-Sud, IN2P3-CNRS, Orsay, France

${ }^{28}$ LLR, Ecole Polytechnique, IN2P3-CNRS, Palaiseau, France

${ }^{29}$ LPNHE, Universités Paris VI and VII, IN2P3-CNRS, Paris, France

${ }^{30}$ Faculty of Science, University of Montenegro, Podgorica, Serbia and Montenegro ${ }^{e}$

${ }^{31}$ Institute of Physics, Academy of Sciences of the Czech Republic, Praha, Czech Republic ${ }^{h}$

${ }^{32}$ Faculty of Mathematics and Physics, Charles University, Praha, Czech Republic ${ }^{h}$

${ }^{33}$ Dipartimento di Fisica Università di Roma Tre and INFN Roma 3, Roma, Italy

${ }^{34}$ Institute for Nuclear Research and Nuclear Energy, Sofia, Bulgaria ${ }^{e}$

${ }^{35}$ Paul Scherrer Institut, Villigen, Switzerland

${ }^{36}$ Fachbereich C, Universität Wuppertal, Wuppertal, Germany

${ }^{37}$ Yerevan Physics Institute, Yerevan, Armenia

${ }^{38}$ DESY, Zeuthen, Germany

${ }^{39}$ Institut für Teilchenphysik, ETH, Zürich, Switzerland ${ }^{i}$ 
${ }^{40}$ Physik-Institut der Universität Zürich, Zürich, Switzerland ${ }^{i}$

${ }^{41}$ Also at Physics Department, National Technical University, Zografou Campus, GR-15773 Athens, Greece

${ }^{42}$ Also at Rechenzentrum, Universität Wuppertal, Wuppertal, Germany

${ }^{43}$ Also at University of P.J. Šafárik, Košice, Slovak Republic

${ }^{44}$ Also at CERN, Geneva, Switzerland

${ }^{45}$ Also at Max-Planck-Institut für Physik, München, Germany

${ }^{46}$ Also at Comenius University, Bratislava, Slovak Republic

${ }^{a}$ Supported by the Bundesministerium für Bildung und Forschung, FRG, under contract numbers 05 H1 1GUA /1, 05 H1 1PAA /1, 05 H1 1PAB /9, 05 H1 1PEA /6, 05 H1 1VHA /7 and $05 \mathrm{HI} \mathrm{IVHB} / 5$

${ }^{b}$ Supported by the UK Particle Physics and Astronomy Research Council, and formerly by the UK Science and Engineering Research Council

${ }^{c}$ Supported by FNRS-FWO-Vlaanderen, IISN-IIKW and IWT and by Interuniversity Attraction Poles Programme, Belgian Science Policy

${ }^{d}$ Partially Supported by the Polish State Committee for Scientific Research, SPUB/DESY/P003/DZ 118/2003/2005

e Supported by the Deutsche Forschungsgemeinschaft

${ }^{f}$ Supported by VEGA SR grant no. 2/4067/24

${ }^{g}$ Supported by the Swedish Natural Science Research Council

${ }^{h}$ Supported by the Ministry of Education of the Czech Republic under the projects LC527 and INGO-1P05LA259

${ }^{i}$ Supported by the Swiss National Science Foundation

${ }^{j}$ Supported by CONACYT, México, grant 400073-F

${ }^{k}$ Partially Supported by Russian Foundation for Basic Research, grants 03-02-17291 and 04-02-16445 


\section{Introduction}

This paper presents a study of the hadronic final state in deep-inelastic scattering (DIS), ep $\rightarrow$ $e X$, using event shape variables. Event shapes probe the energy-momentum flow and are sensitive to perturbative QCD (the hard scattering) and non-perturbative QCD (hadronisation). Compared to some other hadronic final state observables, such as jet cross sections, which employ a limited part of the phase space only, the event shape variables include the full statistics and, by definition, are rather insensitive to hadronic energy scale uncertainties. With DIS at HERA a wide range of the scale $Q$ is available for analysis in a single experiment, where $Q$ is the four-momentum transferred by the exchanged boson from the electron to the proton.

Studies of event shapes require an appropriate treatment of hadronisation effects, which often are corrected for using phenomenological models built into event generators. Alternatively, these effects have been treated by power corrections $\mathcal{O}(1 / Q)$ as calculated analytically from first principles by extending perturbative methods into the non-perturbative regime [1-3]. Within this framework, the event shapes are described by the strong coupling constant $\alpha_{s}$ and an effective coupling parameter $\alpha_{0}$ for the hadronisation corrections. Measurements of event shapes thus represent a sensitive test of the power correction approach, and they allow $\alpha_{s}$ to be determined.

Previous analyses of mean values of event shape variables in DIS, as performed by the H1 [4] and ZEUS [5] collaborations at HERA, support the power correction approach. An observed large spread of values obtained for $\alpha_{s}$, however, indicates that higher-order QCD corrections cannot be neglected. Support for power corrections as an appropriate description of the hadronisation is also provided by analyses of mean values and distributions of event shape variables measured in $e^{+} e^{-}$experiments [6-8] .

Early studies of event shape distributions in deep inelastic scattering revealed that the fixedorder QCD calculations available at the time were insufficient for describing the data [9]. Higher-order corrections have since become available in the form of soft gluon resummed calculations matched to NLO matrix elements [10]. This puts the study of event shape distributions and of the interplay between perturbative and non-perturbative QCD in the description of the hadronic final state on a new quantitative level.

In this paper the distributions of five event shape variables are studied. A QCD analysis is performed based on the resummed and matched calculations supplemented by power corrections. This leads to determinations of the power correction coefficient $\alpha_{0}$ and of $\alpha_{s}$ and its dependence on the scale $Q$.

\section{Event Shape Variables}

The aim of this analysis is to study event shape variables within QCD as a function of the relevant hard scale, which in DIS is taken to be the four-momentum $Q$ of the exchanged boson. In addition to the hadronic flow from the hard scattering there are also hadrons in the final state stemming from the proton dissociation, which occurs at much lower scales of about the proton mass. Therefore it is necessary to separate this proton remnant from the hard scattering part of the event. 


\subsection{The Breit Frame}

In the quark parton model the separation of the proton remnant from the hard scattering is clearest in the Breit frame of reference, defined by $2 x \vec{p}+\vec{q}=0$, where $x$ is the Bjorken scaling variable, $\vec{p}$ the momentum of the proton and $\vec{q}$ the momentum of the exchanged boson. The $z$ axis in the Breit frame is defined to coincide with the proton-boson axis, the proton moving in the $+z$ direction. Particles from the remnant are almost collinear to the proton direction, hence the hemisphere defined by pseudorapidity ${ }^{1} \eta>0$ is labelled the remnant hemisphere. In contrast, in the quark parton model the struck quark populates only the current hemisphere $(\eta<0)$.

Higher order processes generate transverse momenta in the final state and may even lead to particles from the hard subprocess leaking into the remnant hemisphere. Still, without knowledge of the detailed structure of the hadronic final state, the Breit frame allows for optimal separation of the current region from the proton remnant. All event shapes referred to in this paper are defined using particles in the current hemisphere only.

The kinematic quantities needed to perform the Breit frame transformation are calculated using the electron-sigma method $[11,12]$. The virtuality $Q^{2}$ of the exchanged boson is reconstructed using the energy and polar angle of the scattered electron, and the inelasticity $y$ is determined employing in addition the energy and longitudinal momentum of all hadronic objects measured in the laboratory frame. This method results in good experimental resolution and is relatively insensitive to initial state QED bremsstrahlung.

\subsection{Definition of Event Shape Variables}

The event shape variables studied in this paper are those for which the calculations of power corrections are available. They are defined as follows.

The Thrust variable $\tau$ measures the longitudinal momentum components projected onto the boson axis. It is defined as

$$
\tau=1-T \quad \text { with } \quad T=\frac{\sum_{h}\left|\vec{p}_{z, h}\right|}{\sum_{h}\left|\vec{p}_{h}\right|} .
$$

The variable $\tau_{C}$ calculates the Thrust with respect to the direction $\vec{n}_{T}$ which maximises the sum of the longitudinal momenta of all particles in the current hemisphere along this axis. It is defined as

$$
\tau_{C}=1-T_{C} \quad \text { with } \quad T_{C}=\max _{\vec{n}_{T}} \frac{\sum_{h}\left|\vec{p}_{h} \cdot \vec{n}_{T}\right|}{\sum_{h}\left|\vec{p}_{h}\right|} .
$$

The Jet Broadening $B$ measures the scalar sum of transverse momenta with respect to the boson axis

$$
B=\frac{\sum_{h}\left|\vec{p}_{t, h}\right|}{2 \sum_{h}\left|\vec{p}_{h}\right|} .
$$

\footnotetext{
${ }^{1}$ The pseudorapidity is defi ned as $\eta=-\ln \tan (\theta / 2)$ with $\theta$ the polar angle with respect to the $z$ axis.
} 
The squared Jet Mass $\rho$ is normalised to four times the squared scalar momentum sum in the current hemisphere

$$
\rho=\frac{\left(\sum_{h} E_{h}\right)^{2}-\left(\sum_{h} \vec{p}_{h}\right)^{2}}{\left(2 \sum_{h}\left|\vec{p}_{h}\right|\right)^{2}} .
$$

In the following the symbol $\rho_{0}$ is used, which indicates that in the above definition the hadrons are treated as massless, replacing the energy $E_{h}$ by the modulus of the 3-momentum $\left|\vec{p}_{h}\right|$.

The $C$-Parameter is defined as

$$
C=\frac{3}{2} \frac{\sum_{h, h^{\prime}}\left|\vec{p}_{h}\right|\left|\vec{p}_{h^{\prime}}\right| \cos ^{2} \theta_{h h^{\prime}}}{\left(\sum_{h}\left|\vec{p}_{h}\right|\right)^{2}},
$$

where $\theta_{h h^{\prime}}$ is the angle between particles $h$ and $h^{\prime}$.

In Eq. 1-5 the momenta are defined in the Breit frame and the sums extend over all particles in the current hemisphere.

An event is only accepted if the energy in the current hemisphere exceeds some value $\epsilon_{\text {lim }}$. This is necessary to ensure the infrared and collinear safety of the observables, because higher order processes may lead to event configurations in which the partons are scattered into the remnant hemisphere and the current hemisphere is completely empty, except for arbitrarily soft emissions. In the analysis the events are required to fulfill the condition

$$
\sum_{h} E_{h}>\epsilon_{\lim }=Q / 10
$$

as part of the event shape definitions. The precise value of this cut-off turns out not to be crucial [4].

The event shape variables may be distinguished according to the event axis used. The definitions of $\tau$ and $B$ employ momentum vectors projected onto the boson direction, while the others do not, like their counterparts in $e^{+} e^{-}$reactions. Explicit use of the boson direction implies sensitivity to radiation into the remnant hemisphere through recoil effects on the current quark [10].

Throughout the paper the symbol $F$ will be used as a generic name for any event shape variable. Note that for all variables $F$ tends to zero in the case of quark parton model reactions neglecting hadronisation effects (small values of $F$ correspond to pencil like configurations of the hadronic final state). Theoretical calculations of event shape distributions and means are discussed in section 5 .

\section{Experimental Technique}

The data were collected with the H1 detector at HERA during the years $1995-2000$ and correspond to an integrated luminosity of $\mathcal{L}_{\text {int }}=106 \mathrm{pb}^{-1}$. The collider was operated with electrons or positrons of $E_{e}=27.6 \mathrm{GeV}$ and protons of $E_{p}=820 \mathrm{GeV}$ or $E_{p}=920 \mathrm{GeV}$, yielding centre-of-mass energies $\sqrt{s}$ of $301 \mathrm{GeV}$ and $319 \mathrm{GeV}$, respectively. For the present study, three data samples are used: 
- $e^{+} p, \sqrt{s} \simeq 301 \mathrm{GeV}, \mathcal{L}_{\text {int }}=30 \mathrm{pb}^{-1}(1995-1997) ;$

- $e^{-} p, \sqrt{s} \simeq 319 \mathrm{GeV}, \mathcal{L}_{\text {int }}=14 \mathrm{pb}^{-1}(1998-1999)$;

- $e^{+} p, \sqrt{s} \simeq 319 \mathrm{GeV}, \mathcal{L}_{\text {int }}=62 \mathrm{pb}^{-1}(1999-2000)$.

The identification of neutral current DIS events is based on the reconstruction of an event vertex and of the scattered electron ${ }^{2}$ in the central tracker and the calorimeter.

\subsection{H1 Detector}

A detailed description of the $\mathrm{H} 1$ detector can be found in $[13,14]$. The most important detector components for the present analysis are the liquid argon (LAr) calorimeter and the central tracking system. $\mathrm{H} 1$ uses a right-handed coordinate system with the $z$ axis along the beam direction, the $+z$ or "forward" direction being that of the outgoing proton beam. The polar angle $\theta$ is defined with respect to the $z$ axis.

The LAr sampling calorimeter $\left(4^{\circ} \leq \theta \leq 154^{\circ}\right)$ consists of lead/liquid argon electromagnetic sections and stainless steel/liquid argon sections for the measurement of hadronic energy. An in situ calibration provides energy scales. The electron energy scale uncertainty in the LAr calorimeter varies between $0.7 \%$ and $3 \%$ [15]. The hadronic energy measurement is performed by applying a weighting technique to the electromagnetic and the hadronic components of the energy deposition in order to account for the unequal response to electrons and hadrons. The systematic uncertainty on the hadronic energy scale amounts to $2 \%$. In the backward region $\left(153^{\circ} \leq \theta \leq 177^{\circ}\right)$ energy is detected by a lead/scintillating fibre Spaghetti-type calorimeter [16].

The central tracking system $\left(25^{\circ} \leq \theta \leq 155^{\circ}\right)$ is located inside the LAr calorimeter and consists of drift and proportional chambers. The chambers and calorimeters are surrounded by a superconducting solenoid providing a uniform field of $1.15 \mathrm{~T}$ inside the tracking volume. The scattered electron is identified by associating tracking information with the corresponding electromagnetic cluster in the LAr calorimeter. The electron scattering angle is known within $3 \mathrm{mrad}$.

For the present analysis the hadronic final state is reconstructed from combined objects, built from calorimeter clusters and tracks, using an energy flow algorithm which ensures that no double counting of energy occurs. Compared to clusters alone the combined objects improve the reconstruction of low momentum particles.

\subsection{Event Selection}

Several quality cuts are applied to the data. A scattered electron has to be found in the LAr calorimeter with a reconstructed energy $E_{e}^{\prime}$ exceeding $11 \mathrm{GeV}$, which ensures a trigger efficiency above $98 \%$. The $z$ position of the event vertex has to be reconstructed within $\pm 35 \mathrm{~cm}$

\footnotetext{
${ }^{2} \mathrm{Here}$ and in the following "electron" is used to refer to both electron and positron unless explicitly stated otherwise.
} 
of the nominal position of the interaction point, which reduces contributions from beam induced background. Non-ep background is further reduced by requiring an event timing which matches the HERA bunch crossing. To suppress badly measured events the missing transverse momentum has to be below $15 \mathrm{GeV}$. The total longitudinal energy balance must satisfy $40 \mathrm{GeV}<\sum_{i} E_{i}\left(1-\cos \theta_{i}\right)<70 \mathrm{GeV}$, where the sum runs over all detected particles. This reduces photoproduction background and initial state photon radiation. Such QED radiative effects, backgrounds and poorly reconstructed events are further suppressed by demanding that the calorimetric energy measurement of the lepton be consistent within $10 \%$ with that derived from the double angle method $[17,18]$.

The kinematic region covered by the analysis is defined by ranges of boson virtuality $Q^{2}$ and inelasticity $y$ :

$$
\begin{gathered}
196<Q^{2}<40,000 \mathrm{GeV}^{2}, \\
0.1<y<0.7
\end{gathered}
$$

which are reconstructed using the electron-sigma method [11, 12].

In total about 108, 000 events satisfy the selection criteria. The PYTHIA [19] program is used to estimate the background from photoproduction events, in which a hadron in the LAr calorimeter is misidentified as an electron. This background is found to be negligible in all bins of the various event shape distributions.

\subsection{Correction to the Hadron Level}

The correction of the data for limited detector acceptance uses the simulation program RAPGAP 2.8 [20] with parton showers and string fragmentation as implemented in JETSET [21]. The parton density functions (pdfs) of the proton are taken from the CTEQ5L [22] set. The data are corrected for QED radiation effects using the HERACLES [23] program. Bin-to-bin correction factors are determined from the Monte Carlo event samples passed through a detailed simulation of the $\mathrm{H} 1$ detector and subjected to the same reconstruction and analysis chain as the data.

The effects of limited detector resolution are corrected for in a separate step. In the bins used for the QCD analysis presented in section 5 the purities are typically $30-50 \%$. To correct for the corresponding bin correlations the covariance matrix is determined with an iterative Bayesian unfolding method [24]. The data are unfolded to the level of hadrons in order to compare with resummed calculations supplemented by power corrections for hadronisation. The correction procedure is performed separately for the three data samples.

\subsection{Experimental Uncertainties}

Several studies are carried out to estimate the experimental systematic error by using alternative settings or assumptions in the Monte Carlo programs. The correlations of these changes between bins of an event shape variable are taken into account unless stated otherwise. 
The event kinematics and therefore the boost to the Breit frame of reference depends strongly on the momentum of the reconstructed electron. Thus the electromagnetic energy scale is varied by its uncertainty of $\pm(0.7 \%-3 \%)$, depending on the $z$ position of the electron cluster within the LAr calorimeter. In addition, the polar and azimuthal angles of the electron are changed by $\pm 3 \mathrm{mrad}$ each.

The event shape variables are by definition insensitive to variations of the overall hadronic energy scale. The effects of the uncertainty on the hadronic intercalibration are investigated by shifting the calibration constants of neighbouring calorimeter regions with respect to each other [25]. Since the final event shape distributions average over all calorimeter regions, the resulting uncertainty on the event shape variables is small.

The model dependence of the correction procedure is estimated by replacing RAPGAP with the DJANGOH 1.2 [26] event generator, which employs the colour dipole model of ARIADNE [27] to simulate higher order QCD radiation. The model uncertainty is estimated as the difference between the results obtained with the two Monte Carlo samples. One half of this is treated as uncorrelated between the bins. The other half is assumed to be fully correlated between bins.

An estimate of the possible intrinsic bias from the unfolding procedure is obtained by unfolding Monte Carlo event samples with themselves. The residuum between the correct hadron level and the unfolded result is taken as the unfolding error, which is assumed not to be correlated between the bins of the distributions.

All systematic errors from different sources are added in quadrature, the model uncertainty and the electromagnetic energy scale uncertainty being the largest individual contributions.

\subsection{Combination of Data Sets}

The combination of separately unfolded data sets proceeds in two steps, first combining the positron data from the two different centre-of-mass energies and second combining the positron and electron data. The distributions of the two positron data sets are compatible with each other within errors. They are combined by calculating the luminosity weighted averages for all bins of the distributions.

In general, the event shape distributions of the $e^{+}$and $e^{-}$data are in very good agreement with each other, though some discrepancies are observed at the highest $Q$ scales. Differences in the event shape distributions between the $e^{+}$and $e^{-}$data sets are expected because of $Z$ exchange contributions to the cross section [28]. Unfortunately, only the $\gamma$ exchange component is accounted for in the calculations of event shape distributions which are fitted to the data. The $e^{+}$and $e^{-}$event shape distributions are thus averaged, weighted with the corresponding cross section such that most of the $Z$ contribution cancels. Due to the smaller integrated luminosity, the $e^{-}$data increase the statistical error of the final spectra. However, this effect is partly compensated by the larger cross section for $e^{-} p$ scattering at high $Q$.

The resulting mean values of $Q$ and $x$ are slightly modified by the combination procedure, giving the values listed in Table 1 . The average centre-of-mass energy of the combined set is $\sqrt{s}=316 \mathrm{GeV}$. 


\begin{tabular}{|c||l|l|l|l|l|l|l|}
\hline \# of $Q$ bin & 1 & 2 & 3 & 4 & 5 & 6 & 7 \\
\hline$Q$ Interval/GeV & {$[14,16]$} & {$[16,20]$} & {$[20,30]$} & {$[30,50]$} & {$[50,70]$} & {$[70,100]$} & {$[100,200]$} \\
\hline$\langle Q\rangle / \mathrm{GeV}$ & 14.9 & 17.7 & 23.8 & 36.9 & 57.6 & 80.6 & 115.6 \\
\hline$\langle x\rangle$ & 0.00841 & 0.0118 & 0.0209 & 0.0491 & 0.116 & 0.199 & 0.323 \\
\hline
\end{tabular}

Table 1: $Q$ intervals, mean $Q$ and mean Bjorken $x$ for the seven $Q$ bins of the analysis.

\section{Event Shape Measurements}

The normalised event shape distributions at the hadron level for thrust, jet broadening, jet mass and the $C$-parameter are shown in Figs. 1 and 2 over a wide range of $\langle Q\rangle=15-116 \mathrm{GeV}$. The data points represent integrals over the bins not applying bin centre corrections. Except for the highest $Q$ bins, the precision of the measurements is not statistically limited.

For each variable the shape of the spectra changes considerably with increasing $Q$, becoming narrower and evolving towards low values. The strong $Q$ dependence of these spectra is characteristic of QCD. The results of the fits described in detail below are shown for comparison.

The present paper focuses on differential distributions of the event shape variables. However, in order to allow for a comparison to previous analyses the mean values of these variables are also determined. The results are shown in Fig. 3 as a function of $Q$. A steady decrease of the means with rising $Q$ is observed. The results of the present analysis are in agreement with those previously obtained [4].

\section{QCD Analysis}

\subsection{Phenomenology}

The QCD calculations used here contain a perturbative part (pQCD) dealing with partons and in addition use power corrections (PC) to describe the hadronisation. The perturbative part is made up of two contributions: fixed order terms calculated to next-to-leading order (NLO) in the strong coupling constant and resummed terms in the next-to-leading-logarithmic (NLL) approximation.

The NLO contribution consists of the first two terms of the perturbative expansion in $\alpha_{s}$ and, for any event shape variable $F$, has the form

$$
\frac{1}{\sigma} \frac{\mathrm{d} \sigma^{\mathrm{NLO}}}{\mathrm{d} F}=c_{1}(F, Q) \alpha_{S}\left(\mu_{r}\right)+c_{2}(F, Q) \alpha_{S}^{2}\left(\mu_{r}\right)
$$

where $\mu_{r}$ is the renormalisation scale, chosen to be $Q$. In order for the truncated series to be a good approximation to the exact solution, $\alpha_{s}(Q)$ needs to be small, i.e. $Q$ should be large.

The coefficients $c_{1}$ and $c_{2}$ can be calculated from the matrix elements of the hard scattering and the parton density functions of the proton. The fixed order coefficients in the $\overline{\mathrm{MS}}$ scheme are 

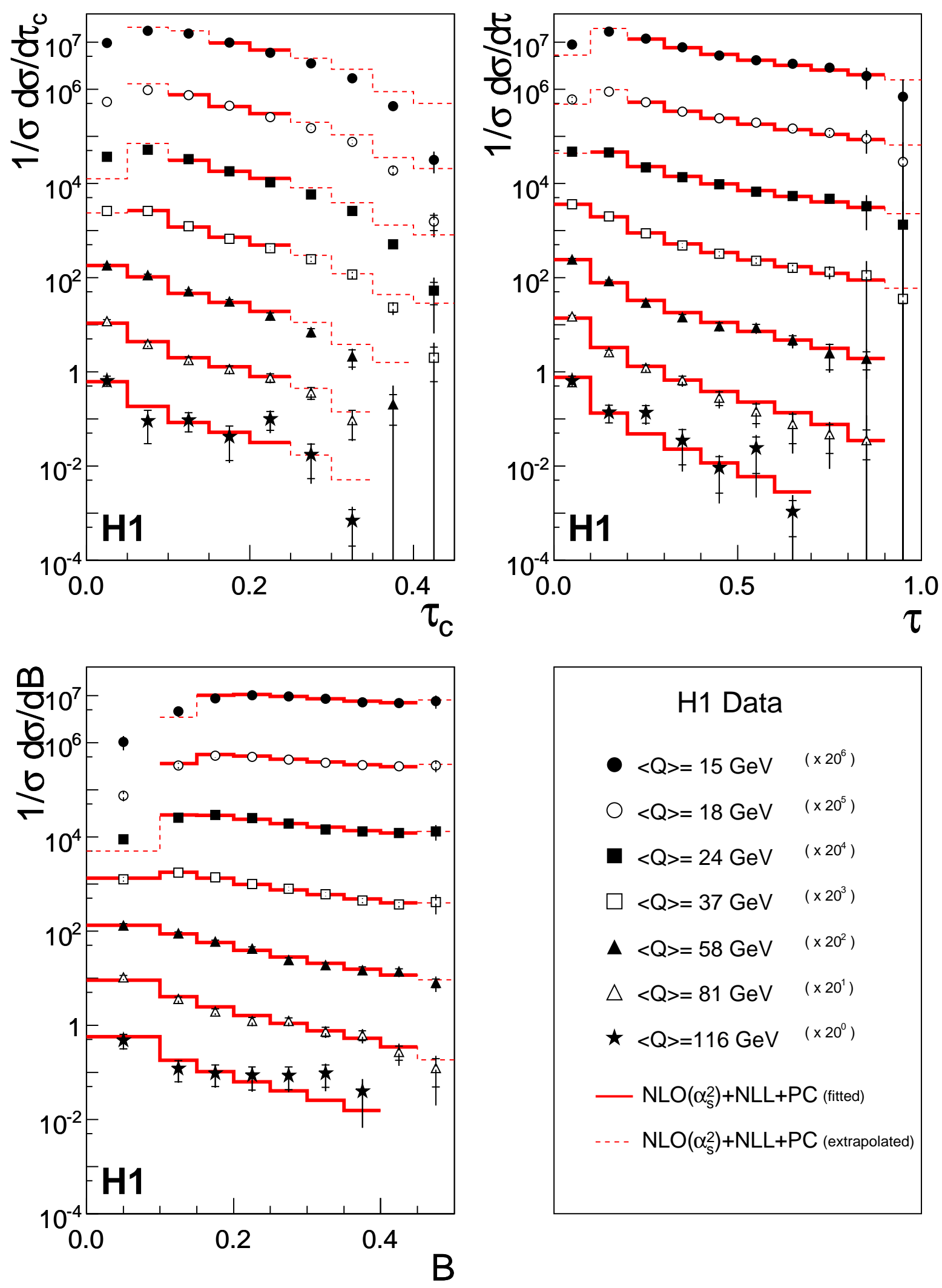

Figure 1: Normalised event shape distributions corrected to the hadron level for $\tau_{C}, \tau$ and $B$. The data are presented with statistical errors (inner bars) and total errors (outer bars). The measurements are compared with fits based on a NLO QCD calculation including resummation (NLL) and supplemented by power corrections (PC). The fit results are shown as solid lines and are extended as dashed lines to those data points which are not included in the QCD fit (see Section 5.3). 

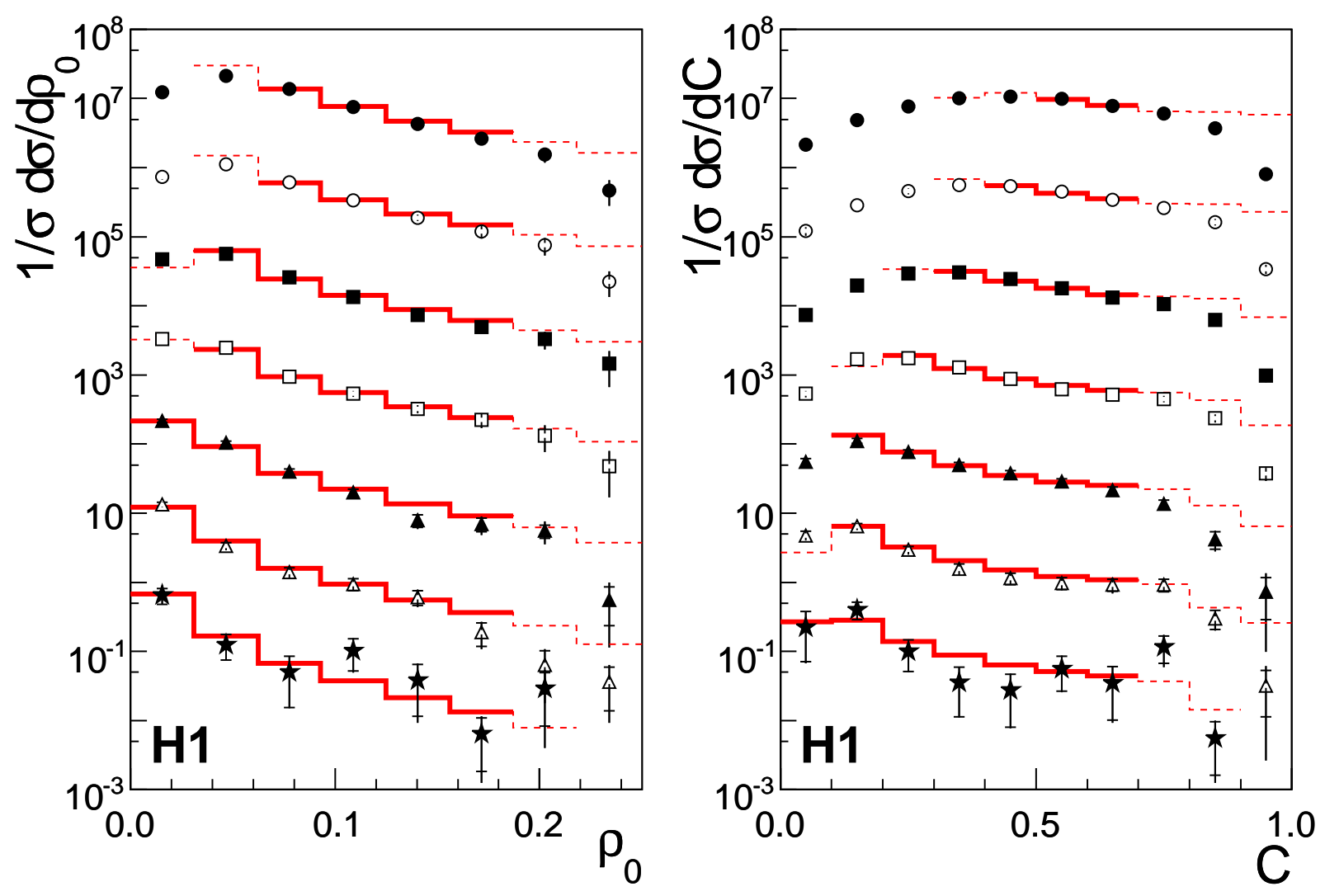

Figure 2: Normalised event shape distributions corrected to the hadron level for $\rho_{0}$ and the $C$-parameter. The data are presented with statistical errors (inner bars) and total errors (outer bars). The measurements are compared with fits based on a NLO QCD calculation including resummation (NLL) and supplemented by power corrections (PC). The fit results are shown as solid lines and are extended as dashed lines to those data points which are not included in the QCD fit (see Section 5.3). The symbols and scale factors are defined in Fig. 1.

determined here using DISASTER++ [29] together with DISPATCH [10]. The parton density functions of the proton are taken from CTEQ5M1 [22].

The region of $F \ll 1$ is dominated by events with soft and/or collinear parton emissions, leading to large perturbative coefficients [30]

$$
c_{n}(F) \underset{F \rightarrow 0}{\sim} \frac{\log ^{2 n-1} F}{F} .
$$

In this region of low $F$ where the bulk of the data lies, the large logarithms need to be resummed to all orders in $\alpha_{s}$. For the event shape variables considered the resummation has been performed by Dasgupta and Salam $[10,31]$ and is available in the DISRESUM package.

To obtain a good description of the data over the full range of $F$, it is necessary to add the fixed order and the resummed calculations, and to subtract any terms which are counted twice, namely the $\mathcal{O}\left(\alpha_{s}\right)$ and $\mathcal{O}\left(\alpha_{s}^{2}\right)$ terms of the resummed result. There are several valid matching schemes available. Comparing the results from the different schemes leads to a residual ambiguity, which is considered in the uncertainty on the prediction. For the central values of 

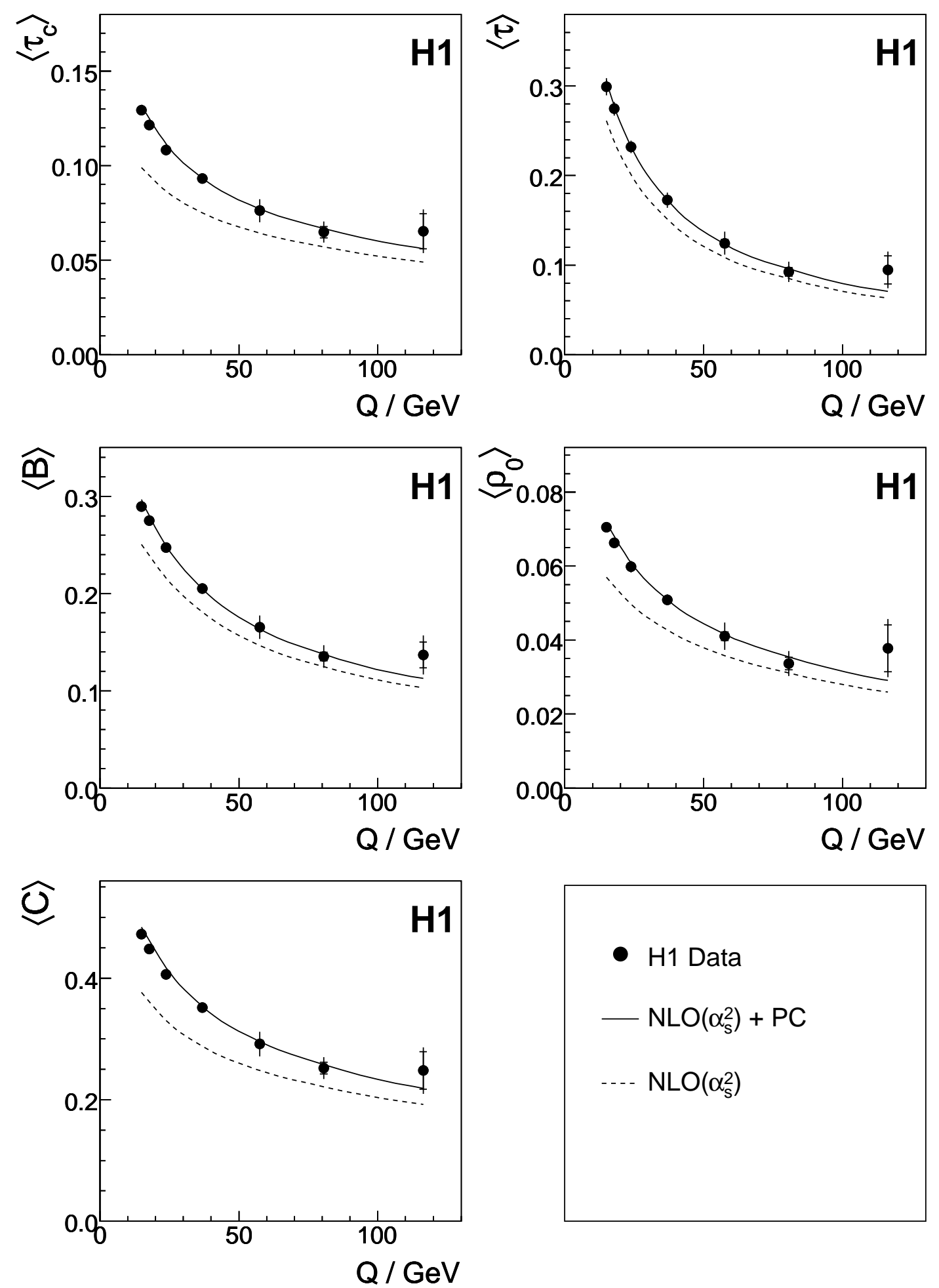

Figure 3: Mean values of event shape variables corrected to the hadron level as a function of the scale $Q$. The data, presented with statistical errors (inner bars) and total errors (outer bars), are compared with the results of NLO QCD fits including power corrections (PC). The dashed curves show the NLO QCD contribution to the fits. 
the present fits the modified $\log \mathrm{R}$ matching scheme is chosen, with the parameter $p_{\mathrm{PT}}$ set to two [10].

All event shape variables are subject to non-perturbative effects due to hadronisation, for which calculations based on partons need to be corrected. The corrections can be determined by using fragmentation models as applied in Monte Carlo programs. For many applications these models lead to a reasonable description of the data. However, the interface between perturbative and non-perturbative processes is not well defined within these models, and there are phenomenological parameters to be tuned.

An alternative approach has been developed [2] based on the observation that non-perturbative corrections are in general suppressed by powers of $(1 / Q)$. For the present event shape variables the leading corrections are proportional to $1 / Q$ according to [2]. The power law behaviour of the corrections can be described by introducing an effective coupling $\alpha_{\text {eff }}$, which is valid for low scales. In the perturbative region the effective coupling has to coincide with the renormalised coupling $\alpha_{s}(Q)$. This is conventionally achieved by matching $\alpha_{\text {eff }}$ to $\alpha_{s}$ at a scale $\mu_{I}=2 \mathrm{GeV}$. This ansatz results in only one single non-perturbative parameter $\alpha_{0}=$ $\mu_{I}^{-1} \int_{0}^{\mu_{I}} \alpha_{\text {eff }}(k) \mathrm{d} k$, being the first moment of the effective coupling integrated over the low scale region up to the matching scale $\mu_{I}$. Power corrections for event shape variables in DIS have been calculated to one-loop [32] and two-loop [33] accuracy.

For the differential distributions the power correction results in a shift of the perturbatively calculated distribution [3]

$$
\frac{1}{\sigma} \frac{\mathrm{d} \sigma(F)}{\mathrm{d} F}=\frac{1}{\sigma} \frac{\mathrm{d} \sigma^{\mathrm{pQCD}}\left(F-a_{F} \mathcal{P}\right)}{\mathrm{d} F},
$$

where $a_{F}$ is of order one and can be calculated perturbatively. For the jet broadening a squeezing is applied in addition to the shift, which is absorbed in the coefficient $a_{F}$ [31].

The power correction term $\mathcal{P}$ is assumed to be universal for all event shape variables. It is proportional to $1 / Q$ and evaluated to be

$$
\mathcal{P}=\frac{16}{3 \pi} \mathcal{M} \frac{\mu_{I}}{Q}\left[\alpha_{0}\left(\mu_{I}\right)-\alpha_{s}(Q)-\frac{\beta_{0}}{2 \pi}\left(\ln \frac{Q}{\mu_{I}}+\frac{K}{\beta_{0}}+1\right) \alpha_{s}^{2}(Q)\right],
$$

where $\beta_{0}=11-2 n_{f} / 3, K=67 / 6-\pi^{2} / 2-5 n_{f} / 9$, and $n_{f}=5$ is the number of active flavours. The so-called Milan factor $\mathcal{M} \simeq 1.05$ ensures the universality at the two-loop level [33].

The simple shift in Eq. 9 cannot be valid over the whole spectrum. At low values of $F$ it may be applied only for $F \gg a_{F} \mathcal{P} \sim \mu_{I} / Q$ [3]. Moreover, at large values of $F$ higher order corrections are substantial and the NLO calculation is not reliable.

The mean value of an event shape variable is modified through non-perturbative effects by an additive constant [1]

$$
\langle F\rangle=\langle F\rangle^{\mathrm{pQCD}}+a_{F} \mathcal{P},
$$

with the same coefficient $a_{F}$ and the same function $\mathcal{P}$ as for the distribution (Eq. 10).

The theory predicts a universal value of $\alpha_{0}\left(\mu_{I}\right)$ of about 0.5 [1]. For mean event shape values this prediction has been confirmed within $20 \%$ in DIS [4] as well as in $e^{+} e^{-}$annihilation [7]. Similar conclusions were drawn in an analysis of differential distributions in $e^{+} e^{-}$ annihilation [7]. 


\subsection{Fit Procedure}

Fits for $\alpha_{s}$ and $\alpha_{0}$ are performed via a $\chi^{2}$ minimisation using MINUIT [34], which for one event shape variable is defined as

$$
\chi^{2}=\sum_{i, j} \Delta_{i} V_{i j}^{-1} \Delta_{j}, \quad \Delta_{i}=m_{i}-t_{i}\left(\alpha_{s}, \alpha_{0}\right)
$$

with $V$ the covariance matrix, $i$ and $j$ extending over the bins included in the fit, $m$ the measured data points and $t\left(\alpha_{s}, \alpha_{0}\right)$ the theory prediction, which depends on the free parameters. The covariance matrix $V$ consists of the sum of the individual covariance matrices from the different sources presented in section 3.4:

$$
V=V_{\text {stat }}+V_{\text {elm.escale }}+V_{\text {had.escale }}+V_{\text {e.track }}+V_{\text {model }}+V_{\text {unfold }},
$$

where $V_{\text {stat }}$ is determined by the unfolding procedure. Only the unfolding and part of the model uncertainties are treated as uncorrelated, i.e. these matrices are diagonal. Summing the covariance matrices in this way corresponds to adding the errors in quadrature. The correlated part of the model uncertainty is determined as half of the difference in the fitted parameters when the fit is repeated, using the program DJANGOH instead of RAPGAP for unfolding.

\subsection{Fits to Spectra}

In order to fit the calculations described in section 5.1 to the measured distributions, the range of the distributions to be used has to be specified. The upper bounds in $F$ are given in [10] and are motivated by properties of the perturbative calculations. The lower bounds in $F$ are set by the behaviour of the power correction, which limits the reliability of the prediction at low $Q$, see Eqs. 9-10. The present analysis makes an effort to extend the fit interval to values of $F$ as low as possible.

For a given event shape, the $\chi^{2}$ is first calculated using the highest $F$ and $Q$ bins only. Bins corresponding to lower $F$ values and lower scales are successively included provided that the $\chi^{2}$ of the fit does not increase by more than four units for each additional bin.

The data are well described by the resummed pQCD calculation supplemented by power corrections as shown in Figs. 1 and 2. In many cases even the lowest $F$ bin could be included in the fit, which is reasonable since, although the distribution diverges as $F \rightarrow 0$, the integral within the measured low $F$ bin remains finite. At low $Q$ values the agreement between the measurements and the calculation degrades. In this domain the hadronisation effects become more important and the simple shift of Eq. 9 is not expected to hold.

The results of the combined fit for $\alpha_{0}$ and $\alpha_{s}\left(m_{Z}\right)$ are displayed in Fig. 4 and summarised in Table 2. The quality of the fits, expressed in terms of $\chi^{2}$ per degree of freedom, is found to be reasonable. For all event shape variables, consistent values for $\alpha_{s}\left(m_{Z}\right)$ and $\alpha_{0}$ are found, with a maximum difference of about two standard deviations between $\tau$ and $C$. A strong negative correlation between $\alpha_{s}\left(m_{Z}\right)$ and $\alpha_{0}$ is observed for all variables. The values of the strong coupling $\alpha_{s}\left(m_{Z}\right)$ are in good agreement with the world average [35], shown for comparison as 


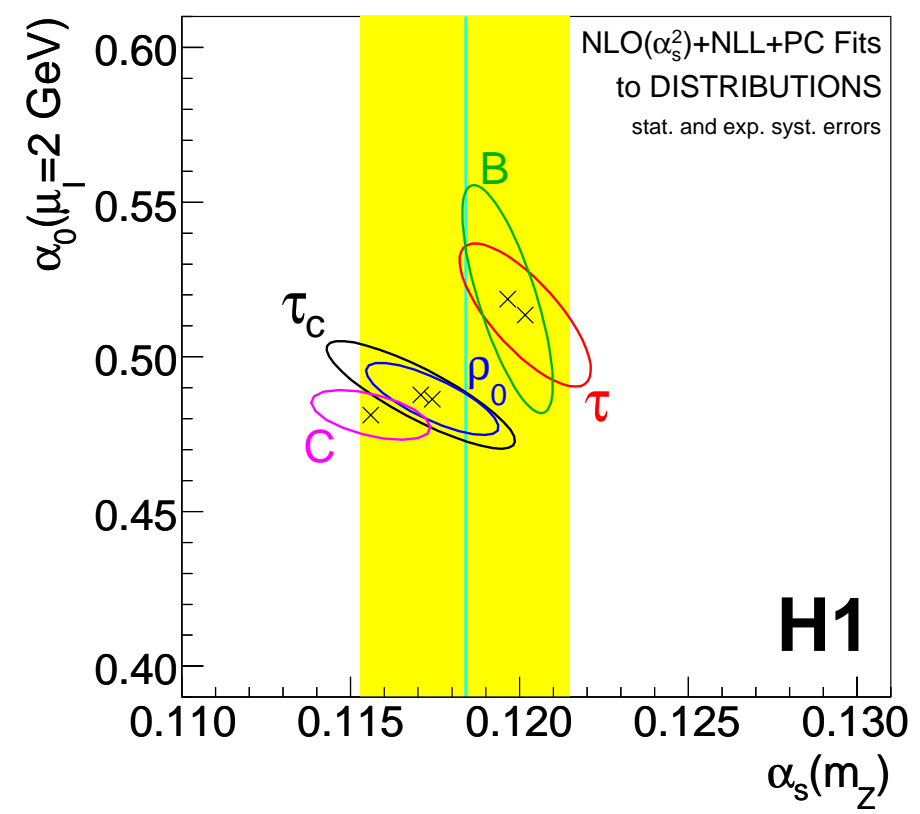

Figure 4: Fit results to the differential distributions of $\tau, B, \rho_{0}, \tau_{C}$ and the $C$-parameter in the $\left(\alpha_{s}, \alpha_{0}\right)$ plane. The $1 \sigma$ contours correspond to $\chi^{2}=\chi_{\min }^{2}+1$, including statistical and experimental systematic uncertainties. The value of $\alpha_{s}$ (vertical line) and its uncertainty (shaded band) are taken from [35].

the shaded band. The non-perturbative parameter $\alpha_{0} \simeq 0.5$ is confirmed to be universal within $10 \%$.

The theoretical uncertainties on the fitted values of $\alpha_{0}$ and $\alpha_{s}\left(m_{Z}\right)$ are determined from the changes to the results under variation in the procedure as follows:

- bins with lower boundaries at $F=0$ are omitted;

- the renormalisation scale $\mu_{r}$ is varied from $Q / 2$ to $2 Q$;

- the infrared matching scale $\mu_{I}$ is varied from $1.5 \mathrm{GeV}$ to $2.5 \mathrm{GeV}$;

- the CTEQ proton pdfs are replaced by three versions of the MRST2001 set [36], which differ in $\alpha_{s}\left(m_{Z}\right)$ from 0.117 to 0.121 ;

- instead of $\log R$ the modified $M$ and modified $M^{2}$ matching schemes [10] are used.

The fit procedure is repeated for each of these variations separately.

All fit results, including the individual contributions to the total error, are given in numerical form in Table 2. The theoretical error is the dominant contribution to the total uncertainty and arises mainly due to the renormalisation scale uncertainty. 


\begin{tabular}{|c|c|c|c|c|c|}
\hline \multicolumn{6}{|c|}{ strong coupling constant $\alpha_{s}\left(m_{Z}\right)$} \\
\hline event shape variable & $\tau_{c}$ & $\tau$ & $B$ & $\rho_{0}$ & $C$ \\
\hline central value & 0.1171 & 0.1202 & 0.1196 & 0.1174 & 0.1156 \\
\hline \multicolumn{6}{|l|}{ uncertainties: } \\
\hline total & $\begin{array}{l}+0.0068 \\
-0.0062 \\
\end{array}$ & $\begin{array}{l}+0.0072 \\
-0.0058 \\
\end{array}$ & $\begin{array}{c}+0.0072 \\
{ }_{-0}^{0.0064} \\
\end{array}$ & $\begin{array}{l}+0.0070 \\
-0.0056 \\
\end{array}$ & $\begin{array}{l}+0.0073 \\
-0.0054 \\
\end{array}$ \\
\hline total experimental & \pm 0.0035 & \pm 0.0021 & \pm 0.0014 & \pm 0.0021 & \pm 0.0021 \\
\hline statistical experimental & \pm 0.0014 & \pm 0.0006 & \pm 0.0004 & \pm 0.0010 & \pm 0.0009 \\
\hline systematic experimental & \pm 0.0033 & \pm 0.0020 & \pm 0.0013 & \pm 0.0019 & \pm 0.0019 \\
\hline total theoretical & $\begin{array}{l}{ }_{-0.0051}^{+0.0058} \\
{ }_{0}\end{array}$ & $\begin{array}{l}{ }_{-0.0054}^{+0.0068} \\
{ }_{0}\end{array}$ & $\begin{array}{l}{ }_{-0.0063}^{+0.0071} \\
\end{array}$ & $\begin{array}{l}{ }_{-0.0052}^{+0.0067} \\
{ }_{0}\end{array}$ & $\begin{array}{l}{ }_{-0.0049}^{+0.0069} \\
{ }_{0}\end{array}$ \\
\hline$\mu_{r}$ dependence & $\begin{array}{l}+0.0054 \\
-0.0048\end{array}$ & $\begin{array}{l}+0.0058 \\
{ }_{-0.0043}\end{array}$ & $\begin{array}{l}+0.0056 \\
{ }_{-0.0044}\end{array}$ & $\begin{array}{l}+0.0064 \\
-0.0050\end{array}$ & $\begin{array}{l}+0.0069 \\
-0.0048\end{array}$ \\
\hline$\mu_{I}$ dependence & $\begin{array}{l}+0.0002 \\
{ }_{-0.0002}\end{array}$ & $<10^{-4}$ & $<10^{-4}$ & $\begin{array}{l}+0.0002 \\
{ }_{-0.0002}\end{array}$ & $<10^{-4}$ \\
\hline fit interval & $\begin{array}{l}+0.0015 \\
{ }_{-0.0018}^{0.0018}\end{array}$ & $\begin{array}{l}+0.0007 \\
{ }_{-0.0022}^{+}\end{array}$ & $\begin{array}{l}+0.0001 \\
{ }_{-0.0009}\end{array}$ & $\begin{array}{l}+0.0010 \\
+0.0007\end{array}$ & $\begin{array}{l}+0.0003 \\
{ }_{-0.0004}\end{array}$ \\
\hline parton density functions & $\begin{array}{l}+0.0002 \\
{ }_{-0.0001}\end{array}$ & $\begin{array}{l}+0.0003 \\
{ }_{-0.0010}\end{array}$ & $\begin{array}{l}+0.0006 \\
{ }_{-0.0007}\end{array}$ & $\begin{array}{l}+0.0001 \\
{ }_{-0.0002}\end{array}$ & $\begin{array}{l}+0.0002 \\
{ }_{-0.0001}\end{array}$ \\
\hline matching scheme & $\begin{array}{l}+0.0015 \\
+0.0005\end{array}$ & $\begin{array}{l}+0.0036 \\
+0.0022\end{array}$ & $\begin{array}{l}+0.0043 \\
+0.0043\end{array}$ & $\begin{array}{l}+0.0018 \\
+0.0009\end{array}$ & $\begin{array}{l}-0.0005 \\
-0.0009\end{array}$ \\
\hline
\end{tabular}

\begin{tabular}{|c|c|c|c|c|c|}
\hline \multicolumn{6}{|c|}{ non perturbative coupling $\alpha_{0}\left(\mu_{I}=2 \mathrm{GeV}\right)$} \\
\hline event shape variable & $\tau_{c}$ & $\tau$ & $B$ & $\rho_{0}$ & $C$ \\
\hline central value & 0.488 & 0.513 & 0.519 & 0.486 & 0.481 \\
\hline \multicolumn{6}{|l|}{ uncertainties: } \\
\hline total & $\begin{array}{l}+0.037 \\
-0.035 \\
\end{array}$ & $\begin{array}{l}0.034 \\
-0.039 \\
\end{array}$ & $\begin{array}{l}+0.059 \\
-0.049 \\
\end{array}$ & $\begin{array}{l}+0.023 \\
-0.035 \\
\end{array}$ & $\begin{array}{r}+0.028 \\
-0.042 \\
\end{array}$ \\
\hline total experimental & \pm 0.021 & \pm 0.025 & \pm 0.039 & \pm 0.014 & \pm 0.008 \\
\hline statistical experimental & \pm 0.009 & \pm 0.009 & \pm 0.006 & \pm 0.006 & \pm 0.005 \\
\hline systematic experimental & \pm 0.019 & \pm 0.023 & \pm 0.038 & \pm 0.013 & \pm 0.007 \\
\hline total theoretical & $\begin{array}{l}+0.030 \\
{ }_{-0.027}^{+}\end{array}$ & $\begin{array}{l}+0.022 \\
-0.029\end{array}$ & $\begin{array}{l}+0.044 \\
{ }_{-0.029}^{+0.029}\end{array}$ & $\begin{array}{l}+0.019 \\
-0.032 \\
\end{array}$ & $\begin{array}{l}+0.026 \\
-0.041\end{array}$ \\
\hline$\mu_{r}$ dependence & $\begin{array}{l}+0.020 \\
-0.026 \\
\end{array}$ & $\begin{array}{l}+0.018 \\
-0.027 \\
\end{array}$ & $\begin{array}{l}+0.030 \\
-0.028 \\
\end{array}$ & $\begin{array}{l}+0.017 \\
-0.027 \\
\end{array}$ & $\begin{array}{l}+0.022 \\
-0.038 \\
\end{array}$ \\
\hline fit interval & $\begin{array}{l}+0.022 \\
-0.007\end{array}$ & $\begin{array}{l}+0.008 \\
-0.005 \\
\end{array}$ & $\begin{array}{l}+0.030 \\
+0.006 \\
\end{array}$ & $\begin{array}{l}-0.003 \\
-0.016 \\
\end{array}$ & $\begin{array}{l}+0.006 \\
-0.003 \\
\end{array}$ \\
\hline parton density functions & $\begin{array}{l}+0.001 \\
{ }_{-0.001}\end{array}$ & $\begin{array}{l}+0.006 \\
+0.004\end{array}$ & $\begin{array}{l}+0.011 \\
+0.003\end{array}$ & $\begin{array}{l}+0.001 \\
{ }_{-0.001}\end{array}$ & $\begin{array}{l}+0.001 \\
-0.002\end{array}$ \\
\hline matching scheme & $\begin{array}{l}-0.005 \\
-0.012\end{array}$ & $\begin{array}{l}-0.009 \\
-0.023\end{array}$ & $\begin{array}{l}+0.006 \\
{ }_{-0.010}\end{array}$ & $\begin{array}{l}-0.006 \\
-0.009\end{array}$ & $\begin{array}{l}-0.014 \\
-0.014\end{array}$ \\
\hline
\end{tabular}

\begin{tabular}{|l|c|c|c|c|c|}
\hline correlation coefficient $\alpha_{s}, \alpha_{0}$ & -0.85 & -0.76 & -0.75 & -0.78 & -0.51 \\
\hline$\chi^{2} /$ d.o.f. (experimental errors) & 1.13 & 0.51 & 0.81 & 1.40 & 1.20 \\
\hline
\end{tabular}

Table 2: Results of simultaneous fits of $\alpha_{s}\left(m_{Z}\right)$ and $\alpha_{0}\left(\mu_{I}=2 \mathrm{GeV}\right)$ to the distributions of the event shape variables $\tau_{C}, \tau, B, \rho_{0}$ and $C$. The statistical and experimental systematic errors as well as the theoretical uncertainties are given.

The good agreement of the results for all event shape variables allows a common set of values of $\alpha_{s}\left(m_{Z}\right)$ and $\alpha_{0}$ to be derived by applying an averaging procedure to the results from the individual event shape variables. In this procedure, the $\chi^{2}$ minimisation takes into account 
all experimental and theoretical errors and the correlations between $\alpha_{s}\left(m_{Z}\right)$ and $\alpha_{0}$ as given in Table 2. In addition the correlations among the observables are considered. The precision on the very large correlation coefficients within the group $\{\tau, B\}$ and the group $\left\{\tau_{C}, \rho_{o}, C\right\}$ is not sufficient to allow the correlation matrix to be used directly in a $\chi^{2}$ minimisation. Instead the averaging is performed in two steps. Firstly, the results within these two groups are combined, neglecting the correlations among the observables. The smallest uncertainty of the contributing measurements is conservatively taken as the uncertainty of the group average. Secondly, the two group averages, being only moderately correlated, are combined using the corresponding correlation matrix.

The averaging procedure results in:

$$
\begin{aligned}
\alpha_{s}\left(m_{Z}\right) & =0.1198 \pm 0.0013(\exp )_{-0.0043}^{+0.0056}(\text { theo }) \\
\alpha_{0} & =0.476 \pm 0.008(\exp )_{-0.059}^{+0.018}(\text { theo })
\end{aligned}
$$

with a fit quality of $\chi^{2} /$ d.o.f. $=4.9 / 2$. Here the theoretical error is derived from the renormalisation scale uncertainty. Note that the combined value of $\alpha_{0}$ is lower than the individual values, due to the negative correlations between $\alpha_{0}$ and $\alpha_{s}\left(m_{Z}\right)$.

If instead the correlations between the two groups of event shape observables are neglected, a consistent result is obtained.

\subsection{Running of Strong Coupling $\alpha_{s}(Q)$}

In the previous section it was shown that the concept of power corrections provides a good description of hadronisation effects in the differential event shape distributions. Alternatively, one may assume the validity of the power corrections and investigate the scale dependence of the strong coupling $\alpha_{s}(Q)$. For each event shape variable a $Q$ independent $\alpha_{0}$ parameter and an $\alpha_{s}(Q)$ for each $Q$ bin are fitted.

The fitted values of $\alpha_{s}(Q)$ are presented in Fig. 5. The running of the strong coupling is clearly observed for each of the event shape variables over a wide scale range between $\langle Q\rangle=$ $15 \mathrm{GeV}$ and $\langle Q\rangle=116 \mathrm{GeV}$. The numerical values with experimental errors are given in Table 3. The theoretical uncertainties are of similar size to those given in Table 2 .

The fit results of the different event shapes are compatible with each other and again may be combined. The correlations among the observables are taken into account in a two-step procedure as described in the previous section. The averaged $\alpha_{s}(Q)$ values are displayed as a function of $Q$ in Fig. 6 and listed in Table 3. A fit of the renormalisation group equation to the measured $\alpha_{s}(Q)$ yields

$$
\alpha_{s}\left(m_{Z}\right)=0.1178 \pm 0.0015(\exp )_{-0.0061}^{+0.0081}(\text { theo }),
$$

with $\chi^{2} / \mathrm{ndf}=8.3 / 6$. This value is in good agreement with the two-parameter fit result quoted in Eq. 14, though the scale uncertainty is somewhat larger here. The difference with respect to the result quoted in Eq. 14 is that here individual power correction parameters $\alpha_{0}$ are associated to each observable. 


\begin{tabular}{|l||c|c|c|c|c|c|}
\hline \multicolumn{1}{|l||}{$\begin{array}{l}Q / \\
\mathrm{GeV}\end{array}$} & $\tau_{c}$ & $\tau$ & $B$ & $\rho_{0}$ & $C$ & $\left\langle\alpha_{s}(Q)\right\rangle$ \\
\cline { 2 - 7 } & \multicolumn{7}{c|}{ strong coupling constant $\boldsymbol{\alpha}_{\boldsymbol{s}}(\boldsymbol{Q})$} \\
\hline \hline 15 & $0.155 \pm 0.007$ & $0.171 \pm 0.008$ & $0.164 \pm 0.004$ & $0.162 \pm 0.006$ & $0.162 \pm 0.006$ & $0.163 \pm 0.004$ \\
18 & $0.149 \pm 0.007$ & $0.165 \pm 0.005$ & $0.160 \pm 0.003$ & $0.158 \pm 0.005$ & $0.157 \pm 0.006$ & $0.159 \pm 0.003$ \\
24 & $0.150 \pm 0.005$ & $0.148 \pm 0.006$ & $0.151 \pm 0.003$ & $0.147 \pm 0.004$ & $0.147 \pm 0.004$ & $0.148 \pm 0.003$ \\
37 & $0.133 \pm 0.006$ & $0.139 \pm 0.004$ & $0.141 \pm 0.003$ & $0.137 \pm 0.004$ & $0.130 \pm 0.004$ & $0.136 \pm 0.003$ \\
58 & $0.128 \pm 0.006$ & $0.127 \pm 0.005$ & $0.129 \pm 0.005$ & $0.127 \pm 0.006$ & $0.131 \pm 0.006$ & $0.128 \pm 0.005$ \\
81 & $0.110 \pm 0.005$ & $0.107 \pm 0.009$ & $0.108 \pm 0.006$ & $0.110 \pm 0.006$ & $0.104 \pm 0.006$ & $0.108 \pm 0.004$ \\
116 & $0.090 \pm 0.018$ & $0.138 \pm 0.047$ & $0.123 \pm 0.027$ & $0.112 \pm 0.025$ & $0.122 \pm 0.011$ & $0.114 \pm 0.011$ \\
\hline \hline$\alpha_{0}$ & $0.496 \pm 0.014$ & $0.515 \pm 0.020$ & $0.516 \pm 0.034$ & $0.484 \pm 0.011$ & $0.477 \pm 0.007$ & \\
\hline
\end{tabular}

Table 3: Fitted values of $\alpha_{s}(Q)$ as determined from the differential distributions of the event shape variables $\tau_{C}, \tau, B, \rho_{0}$ and $C$ and the averaged values $\left\langle\alpha_{s}(Q)\right\rangle$; the fitted parameters $\alpha_{0}$ are given in the last line.

\subsection{Fits to Mean Values}

The mean values of the event shape variables, presented as a function of the scale $Q$ in Fig. 3, are also subjected to QCD fits. For this application the resummed calculation can not be used, because of difficulties at high values of the event shape variable, which are related to sub-leading logarithms and the matching procedure [28]. These regions are excluded from the fits to the full spectra, but by definition contribute to the mean values. Hence the theoretical prediction for the mean values is solely based on a NLO calculation, supplemented by power corrections.

The results of the fits are displayed in Fig. 3 and the fitted values of $\alpha_{s}\left(m_{Z}\right)$ and $\alpha_{0}$ are shown in Fig. 7. The non-perturbative parameters cluster around a common value of $\alpha_{0} \simeq$ $0.45-0.50$, thus supporting universality at the $10 \%$ level. The fitted values for $\alpha_{s}\left(m_{Z}\right)$ exhibit a rather large spread.

Compared to the results obtained from the event shape distributions shown in Fig. 4, higher values of $\alpha_{s}$ are found using $\tau_{C}, \rho_{0}$ and in particular the $C$-parameter, and a somewhat lower value is obtained from $B$. The analyses of both the event shape distributions and mean values are based on the same data and there is no experimental reason why they should lead to different results. Therefore, it is likely that the different theoretical treatment for mean values is the source of the observed deviations.

A dedicated study of mean values has been published previously by the H1 collaboration [4] for $7<Q<100 \mathrm{GeV}$. As mentioned in section 4 the current and previous measurements are in agreement in the phase space of overlap. The results of both QCD analyses are also consistent with each other. The previous analysis shows a larger sensitivity to the parameters $\alpha_{s}$ and $\alpha_{0}$ because it includes data at lower $Q$. This leads to substantially reduced error ellipses compared to those of Fig. 7, in particular for the $\langle\tau\rangle$ observable.

Differences between sets of QCD parameters $\alpha_{s}$ and $\alpha_{0}$ determined in differential distributions and mean values, similar to those reported here, have been observed in $e^{+} e^{-}$annihilation by DELPHI [7]. Discrepancies have also been found by other experiments [6,8]. In general, similar values of $\alpha_{0} \simeq 0.5$ are found for event shape variables in $e^{+} e^{-}$scattering, but with a much larger spread than in deep-inelastic scattering, the jet broadening results in particular being different from the others. 

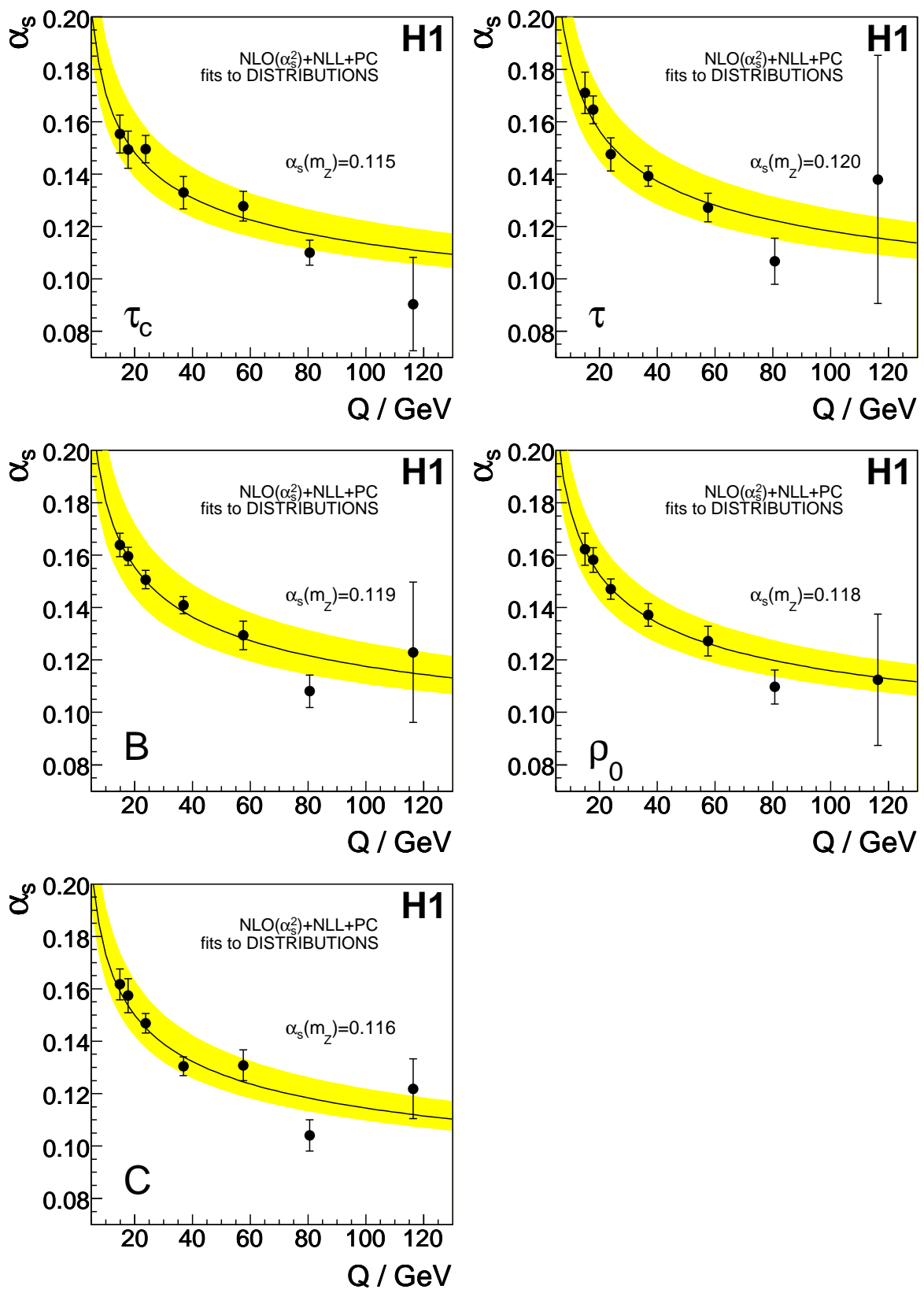

Figure 5: The strong coupling $\alpha_{s}$ as a function of the scale $Q$. The individual fit results, shown as points with error bars, are obtained from fits to the differential distributions in $\tau_{C}, \tau, B, \rho_{0}$ and $C$ within each $Q$ bin. The errors represent the total experimental uncertainties. For each event shape observable a value of $\alpha_{s}\left(m_{Z}\right)$ is indicated in the plot, determined from a fit to the $\alpha_{s}(Q)$ results using the QCD renormalisation group equation. The corresponding fit curves are shown as full lines. The shaded bands represent the uncertainties on $\alpha_{s}(Q)$ from renormalisation scale variations.

\section{Conclusions}

Accurate measurements of event shape variables in deep-inelastic ep scattering are presented based on $106 \mathrm{pb}^{-1}$ of data with four-momentum transfer $Q$ ranging between $14 \mathrm{GeV}$ and 


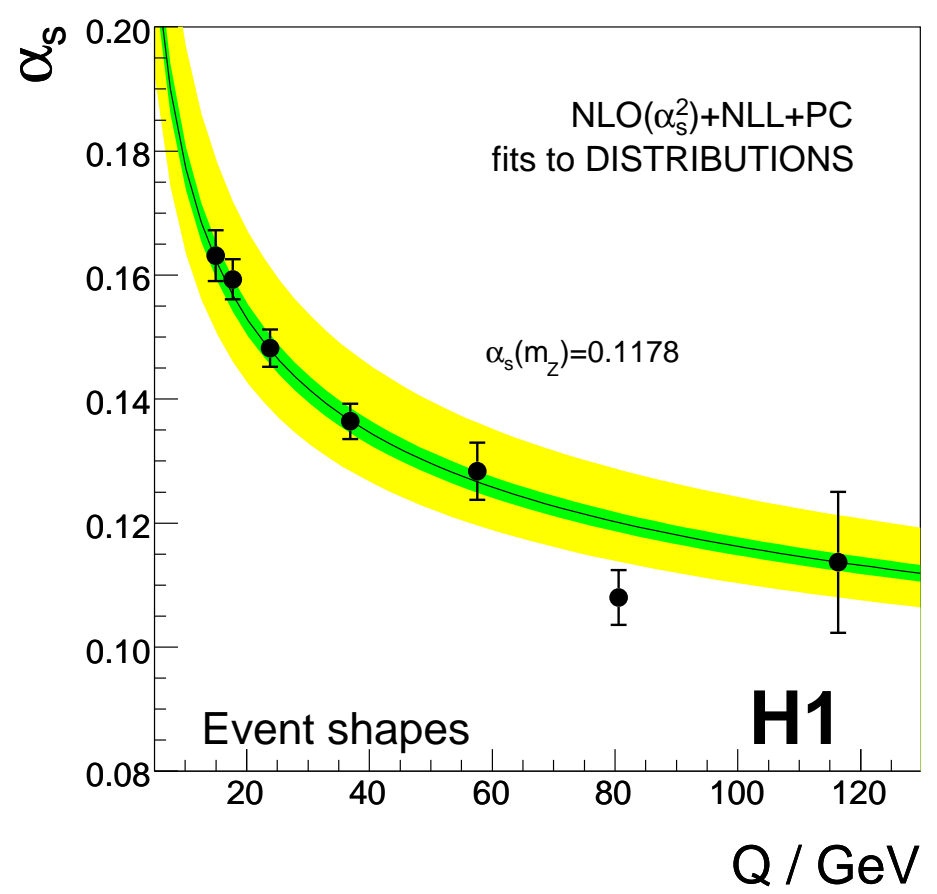

Figure 6: The strong coupling $\alpha_{s}$ as a function of the scale $Q$ from an average of the results obtained by fitting the differential event shape distributions. The errors represent the total experimental uncertainties. A value of $\alpha_{s}\left(m_{Z}\right)$ is indicated in the plot, determined from a fit to the $\alpha_{s}(Q)$ results using the QCD renormalisation group equation. The fit curve is shown as the full line. The inner (outer) shaded band represents the uncertainty of the fitted $\alpha_{s}(Q)$ from experimental errors (the renormalisation scale variation).

$200 \mathrm{GeV}$. Resummed perturbative QCD predictions together with power corrections give good descriptions of the spectra of the observables thrust, jet broadening, jet mass and $C$-parameter. The use of resummed calculations extends the good description to low values of the event shape variables, corresponding to pencil like configurations.

The results of a two-parameter fit of the strong coupling constant $\alpha_{s}$ and the effective nonperturbative coupling $\alpha_{0}$ for the various event shape observables are consistent with each other. The values for $\alpha_{s}$ agree with the world average. The parameter $\alpha_{0}$, which accounts for hadronisation, is consistently found to be 0.5 within $10 \%$, in good agreement with theoretical expectation. A combined analysis of all event shape variables yields

$$
\begin{aligned}
\alpha_{s}\left(m_{Z}\right) & =0.1198 \pm 0.0013(\exp )_{-0.0043}^{+0.0056}(\text { theo }), \\
\alpha_{0} & =0.476 \pm 0.008(\exp )_{-0.059}^{+0.018}(\text { theo }),
\end{aligned}
$$

where the theoretical error is derived from the renormalisation scale uncertainty. Relaxing the requirement of a common value of $\alpha_{0}$, the data are used to investigate the scale dependence of the strong coupling over a wide range of $Q=15-116 \mathrm{GeV}$. The running of $\alpha_{s}(Q)$ is clearly observed for each event shape variable, in accordance with the expected evolution. Combining the results of all variables leads to $\alpha_{s}\left(m_{Z}\right)=0.1178 \pm 0.0015$ (exp) ${ }_{-0.0061}^{+0.0081}$ (theo). The errors 


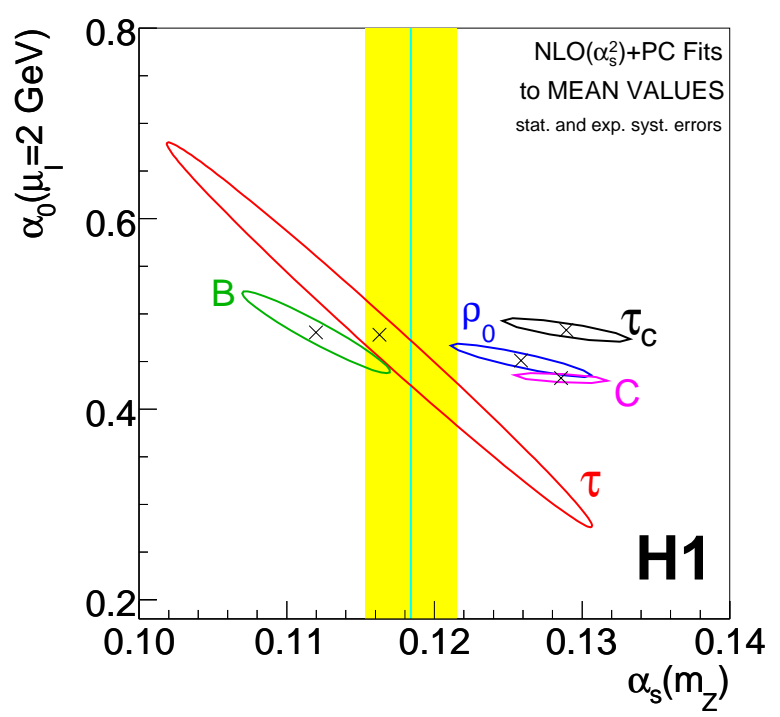

Figure 7: Results of fits to the mean values of $\tau, B, \rho_{0}, \tau_{C}$ and the $C$-parameter in the $\left(\alpha_{s}, \alpha_{0}\right)$ plane. The $1 \sigma$ contours correspond to $\chi^{2}=\chi_{\min }^{2}+1$, including statistical and experimental systematic uncertainties. The value of $\alpha_{s}$ (vertical line) and its uncertainty (shaded band) are taken from [35]. Note the enlarged scale compared to Fig. 4.

are dominated by the renormalisation scale uncertainty, which suggests that missing higher order terms in the perturbative calculation are important.

In the analysis of the event shape means the results for $\alpha_{s}\left(m_{Z}\right)$ and $\alpha_{0}$ are less accurate than those obtained in the fits to the distributions. While the non-perturbative parameters $\alpha_{0}$ cluster again around a common value of 0.5 , the results for the strong coupling $\alpha_{s}$ obtained from the five event shape means exhibit a spread considerably larger than is expected from their individual uncertainties. These discrepancies may be related to an insufficient theoretical treatment, which lacks resummed calculations.

The observed universality of $\alpha_{0}$ for both distributions and mean values of the event shape variables supports the concept of power corrections. Hence, it can be considered as an appropriate alternative to conventional models for the description of hadronisation effects for event shape variables.

\section{Acknowledgments}

We are grateful to the HERA machine group whose outstanding efforts have made this experiment possible. We thank the engineers and technicians for their work in constructing and maintaining the $\mathrm{H} 1$ detector, our funding agencies for financial support, the DESY technical staff for continual assistance and the DESY directorate for support and for the hospitality which they extend to the non DESY members of the collaboration. We want to thank M. Dasgupta and G.P. Salam for many valuable discussions and for good cooperation over the years. 


\section{References}

[1] Y. L. Dokshitzer and B. R. Webber, Phys. Lett. B352, 451-455 (1995), hep-ph/9504219.

[2] Y. L. Dokshitzer, G. Marchesini and B. R. Webber, Nucl. Phys. B469, 93-142 (1996), hep-ph/9512336.

[3] Y. L. Dokshitzer and B. R. Webber, Phys. Lett. B404, 321-327 (1997), hep-ph/9704298.

[4] H1 Collaboration, C. Adloff et al., Eur. Phys. J. C14, 255-269 (2000), hep-ex/9912052.

[5] ZEUS Collaboration, S. Chekanov et al., Eur. Phys. J. C27, 531-545 (2003), hepex/0211040.

[6] P. A. Movilla Fernandez, S. Bethke, O. Biebel and S. Kluth, Eur. Phys. J. C22, 1-15 (2001), hep-ex/0105059.

[7] DELPHI Collaboration, J. Abdallah et al., Eur. Phys. J. C29, 285-312 (2003), hepex/0307048.

[8] ALEPH Collaboration, A. Heister et al., Eur. Phys. J. C35, 457-486 (2004).

[9] H1 Collaboration, C. Adloff et al., Paper No. 530 contributed to 29th International Conference on High-Energy Physics (ICHEP 98), Vancouver, Canada, 1998.

[10] M. Dasgupta and G. P. Salam, JHEP 08, 032 (2002), hep-ph/0208073.

[11] U. Bassler and G. Bernardi, Nucl. Instrum. Meth. A361, 197-208 (1995), hepex/9412004.

[12] U. Bassler and G. Bernardi, Nucl. Instrum. Meth. A426, 583-598 (1999), hepex/9801017.

[13] H1 Collaboration, I. Abt et al., Nucl. Instrum. Meth. A386, 310-347 (1997).

[14] H1 Collaboration, I. Abt et al., Nucl. Instrum. Meth. A386, 348-396 (1997).

[15] H1 Collaboration, C. Adloff et al., Eur. Phys. J. C30, 1-32 (2003), hep-ex/0304003.

[16] R. D. Appuhn et al., Nucl. Instrum. Meth. A386, 397-408 (1997).

[17] K. C. Höger, Proceedings of the Workshop "Physics at HERA", vol. 1, eds. W. Buchmüller and G. Ingelman, DESY (1992) 43.

[18] S. Bentvelsen, J. Engelen and P. Kooijman, NIKHEF-H-92-02.

[19] T. Sjöstrand et al., Comput. Phys. Commun. 135, 238-259 (2001), hep-ph/0010017.

[20] H. Jung, Comp. Phys. Commun. 86, 147-161 (1995).

[21] T. Sjöstrand, Comput. Phys. Commun. 82, 74-90 (1994). 
[22] CTEQ Collaboration, H. L. Lai et al., Eur. Phys. J. C12, 375-392 (2000), hep$\mathrm{ph} / 9903282$.

[23] A. Kwiatkowski, H. Spiesberger and H. J. Möhring, Comp. Phys. Commun. 69, 155-172 (1992).

[24] G. D’Agostini, Nucl. Instrum. Meth. A362, 487-498 (1995).

[25] T. Kluge, PhD Thesis, RWTH Aachen, DESY-THESIS-2004-024 (available at http://www-h1.desy.de/publications/theses Jist.html).

[26] K. Charchula, G. A. Schuler and H. Spiesberger, Comput. Phys. Commun. 81, 381-402 (1994).

[27] L. Lönnblad, Comput. Phys. Commun. 71, 15-31 (1992).

[28] M. Dasgupta and G. P. Salam, private communication.

[29] D. Graudenz, “DISASTER++ version 1.0”, hep-ph/9710244.

[30] R.K. Ellis, W.J. Stirling, B.R. Webber, "QCD and Collider Physics", Cambridge University Press, 1996.

[31] M. Dasgupta and G. P. Salam, Eur. Phys. J. C24, 213-236 (2002), hep-ph/0110213.

[32] M. Dasgupta and B. R. Webber, Eur. Phys. J. C1, 539-546 (1998), hep-ph/9704297.

[33] M. Dasgupta and B. R. Webber, JHEP 10, 001 (1998), hep-ph/9809247.

[34] F. James and M. Roos, Comput. Phys. Commun. 10, 343-367 (1975).

[35] S. Bethke, Nucl. Phys. Proc. Suppl. 135, 345-352 (2004), hep-ex/0407021.

[36] A. D. Martin, R. G. Roberts, W. J. Stirling and R. S. Thorne, Eur. Phys. J. C23, 73-87 (2002), hep-ph/0110215. 